\title{
Paradoxical Prefrontal-Amygdala Recruitment to Angry and Happy Expressions in Pediatric Posttraumatic Stress Disorder
}

\author{
Taylor J Keding' and Ryan J Herringa*, I \\ 'Department of Psychiatry, University of Wisconsin School of Medicine and Public Health, Madison, WI, USA
}

\begin{abstract}
The neural substrates of pediatric posttraumatic stress disorder (PTSD) remain incompletely understood, but likely involve abnormal function and development of emotion processing circuitry. Valence-specific and age-related abnormalities during emotion processing have not been elucidated. We examined implicit emotional face processing in pediatric PTSD, predicting abnormalities specific to threat-related emotion. Youth (ages $8-18$ years) with PTSD $(n=25)$ and healthy youth $(n=28)$ completed a dynamic emotional face task during fMRI, viewing faces changing from neutral to angry or happy, or changing shape control. Group and cross-sectional age-related differences in activation and functional connectivity were examined in amygdala/hippocampus, medial prefrontal cortex (mPFC), and whole-brain analyses. The post hoc analyses examined the relationship of neural abnormalities with symptom measures of PTSD, anxiety, and depression. Compared with decreased activation with age in healthy youth, PTSD youth showed increased amygdala activation to emotional faces with age. In a group by emotion interaction, PTSD youth showed dorsal (d)ACC hyperactivation to happy faces relative to healthy youth, with no difference for angry faces. Connectivity analyses revealed paradoxical coupling in prefrontal-amygdala circuits, including dACC-dorsomedial (dm)PFC, amygdala-dmPFC, and amygdala-ventrolateral (vl)PFC. In each case, PTSD youth showed reduced connectivity to angry faces, but increased connectivity to happy faces, the reverse of healthy youth. Valence-abnormal recruitment was associated with greater symptom severity, implicating a role in trauma-related psychopathology in youth. Notably, impaired recruitment during angry faces and heightened recruitment to happy faces may reflect increased salience and ambiguity of positive emotional expressions in pediatric PTSD. Finally, age-related findings suggest a developmental sensitization of the amygdala across emotional expressions in youth with PTSD. These findings provide novel insights into the underlying pathophysiology of pediatric PTSD, extending beyond abnormal neural responses to canonical threat.
\end{abstract}

Neuropsychopharmacology (2016) 4I, 2903-2912; doi:10.1038/npp.201 6.104; published online 20 July 2016

\section{INTRODUCTION}

Pediatric posttraumatic stress disorder (PTSD) is common, affecting $5 \%$ of youth by age 18 years (McLaughlin et al, 2013). Pediatric PTSD is frequently comorbid with other psychiatric illnesses including depression, anxiety, and substance use disorders (Cohen and Scheeringa, 2009). Although significant progress has been made in understanding the neural substrates of adult PTSD, the corresponding pathophysiology in youth remains incompletely understood. Elucidating neural dysfunction in pediatric PTSD, including potential developmental abnormalities, is vital to improving detection and treatment of the disorder.

Meta-analyses of adult PTSD neuroimaging studies suggest abnormal functioning of circuitry underlying emotion appraisal and regulation, including hyperactivation of the amygdala, and hypoactivation of medial prefrontal (mPFC) regulatory regions, including ventromedial (vm)PFC and

\footnotetext{
*Correspondence: Dr RJ Herringa, Department of Psychiatry, University of Wisconsin School of Medicine and Public Health, 600I Research Park Boulevard, Madison, WI 53719, USA, Tel: +I 6082653610 , Fax: + I 608262 9246, E-mail: herringa@wisc.edu

Received 4 March 2016; revised 29 May 2016; accepted I 5 June 2016; accepted article preview online 22 June 2016
}

dorsomedial (dm)PFC, to negative and threat-related stimuli (Etkin and Wager, 2007; Hayes et al, 2012; Patel et al, 2012). The dorsal anterior cingulate cortex (dACC) also shows functional abnormalities in adult PTSD in response to threatrelated stimuli, although with mixed findings (Etkin and Wager, 2007; Hayes et al, 2012; Patel et al, 2012). Hippocampal findings have been variable, although hypoactivation has been observed during fear extinction tasks (Milad et al, 2009). Functional connectivity findings in adult PTSD have also been mixed, suggesting both greater (Fonzo et al, 2010; Gilboa et al, 2004; St Jacques et al, 2011) and lower (Stevens et al, 2013) amygdala-mPFC connectivity to negative stimuli. Overall, neural models of adult PTSD posit hyperactivation of fearpromoting regions (amygdala, dACC), and hypoactivation of emotion regulatory areas (vmPFC, dmPFC) to negative stimuli (Pitman et al, 2012).

Relatively few studies have examined functional brain abnormalities during emotion processing in pediatric PTSD. In response to trauma-related imagery, a small study revealed rostral (r)ACC hypoactivation, but no differences in amygdala activation, relative to healthy controls (Yang et al, 2004). Our prior report in this sample using traumarelated imagery also revealed no differences in amygdala activation, but showed dACC hyperactivation and reduced 
amygdala-mPFC connectivity in pediatric PTSD (Wolf and Herringa, 2016). During face processing, youth with posttraumatic stress symptoms (PTSS) showed hyperactivation of the amygdala and vmPFC, but no differences in dACC activation across neutral and emotional faces (Garrett et al, 2012). Decreased dmPFC activation to fear faces has been reported in female youth with PTSS, suggesting possible sex differences (Crozier et al, 2014). Finally, PTSS were found to be negatively correlated with amygdala-dmPFC/pre-supplementary motor area (SMA) functional connectivity to fear versus neutral faces (Cisler et al, 2013).

These studies provide initial evidence of prefrontalamygdala dysfunction in pediatric PTSD/PTSS, yet important questions remain. First, it remains unclear whether abnormalities in face processing are emotion specific (eg, to threat), as seen in adult PTSD. Second, little is known regarding functional brain connectivity in pediatric PTSD that can provide important information about network-level dysfunction. Finally, age-related abnormalities in face processing have not been reported in pediatric PTSD, and this may provide important clues about neurodevelopmental divergence. Based on studies in healthy youth, this may be especially true for the development of prefrontal-amygdala (Burghy et al, 2012; Gabard-Durnam et al, 2014; Gee et al, 2013b; Herringa et al, 2013a) and prefrontal-hippocampus function (Herringa et al, 2013a). Furthermore, our prior study in this sample revealed decreased dmPFC activation and amygdala-vmPFC connectivity with age during trauma imagery, suggesting altered neurodevelopment in key emotion processing pathways in pediatric PTSD. Whether similar developmental abnormalities are present during emotional face processing remains unknown.

To address these knowledge gaps, we used functional (f) MRI to examine brain activation and functional connectivity to dynamic emotional faces in a cross-sectional sample of youth with severe PTSD and nontraumatized healthy youth. We predicted that youth with PTSD would display agerelated sensitization of the amygdala based on prior studies of childhood adversity (see, eg, Gee et al, 2013a; Swartz et al, 2015; Herringa et al, 2016), and reduced mPFC activation and amygdala-mPFC connectivity relative to healthy youth. Importantly, we predicted that these abnormalities would be specific to threat-related emotion (angry) but would not be present for happy faces.

\section{MATERIALS AND METHODS}

\section{Participants}

Participant recruitment and assessment have been previously described (Keding and Herringa, 2015; Wolf and Herringa, 2016; Patriat et al, 2016) and are briefly summarized here. A total of 28 medication-free youth with PTSD were recruited from area mental health facilities, whereas 33 nontraumatized healthy youth of comparable age and sex were recruited by local advertisements. Exclusion criteria for youth with PTSD included active suicidality, history of psychotic disorder, bipolar disorder, or OCD; recent (past 4 weeks) substance abuse or dependence; IQ $<70$; unstable medical condition; recent use of psychotropic medication (past 4 weeks; 6 weeks for fluoxetine); MRI contraindication; and possibility of pregnancy in females. Healthy participants were free of any history of mental illness. Participant data were excluded for movement and early scan termination ( $n=3$ PTSD, 5 healthy), leaving 25 youth with PTSD and 28 healthy youth in the final analyses. Written parental consent and youth assent were obtained for all participants. All procedures were approved by the University of Wisconsin Health Sciences institutional review board.

\section{Clinical and Behavioral Assessments}

All participants and their caregivers underwent traumatic events and psychiatric screening by a board-certified, child and adolescent psychiatrist (RJH) with the Kiddie Schedule for Affective Disorders and Schizophrenia (KSADS) (Kaufman et al, 1997). PTSD diagnosis was determined using modified DSM-IV criteria (Cohen et al, 2011) by combination of the KSADS and Clinician-Administered PTSD Scale for Children and Adolescents (CAPS-CA) (Weathers et al, 2001). Additional self/caregiver report of symptoms were obtained for PTSD using the UCLA PTSD Reaction Index (PTSD-RI) (Steinberg et al, 2004), depressive symptoms with the Mood and Feelings Questionnaire (MFQ) (Costello and Angold, 1988), and anxiety symptoms with the Screen for Child Anxiety Related Emotional Disorders (SCARED) (Birmaher et al, 1997). See Supplementary Material for further details on behavioral measures.

\section{Dynamic Face Task}

Participants underwent fMRI while completing a dynamic face task (Supplementary Figure S1) that employs implicit cognitive-emotional processing (Almeida et al, 2011; Herringa et al, 2013b). We used an implicit paradigm to ensure that the task could be successfully completed across the entire age range of our sample, as well as both PTSD and healthy youth. Thus, the chances of group- and age-related effects on fMRI are unlikely to be confounded by performance effects, a concern on tasks with increased cognitive load (intrinsic to many explicit paradigms). Participants were asked to identify the color of a semitransparent overlay atop a changing emotional face (angry, happy) or oval shape (control condition) distractor. Each dynamic face changed from neutral to emotion over a 1-s period. Three blocks, each with 12 faces, were presented pseudorandomly for each emotion condition. Emotional face blocks were interspersed by shape blocks, ensuring no two emotion blocks were presented sequentially. See Supplementary Material for further details.

\section{Image Acquisition and Preprocessing}

Please see Supplementary Material for details on image acquisition. Image processing was performed using AFNI (Cox, 1996) and FSL (Woolrich et al, 2009). T1 structural images were registered to the MNI152, $2 \mathrm{~mm}^{3}$ template with linear and nonlinear warps (FSL FLIRT and FNIRT). Functional data were slice-time and motion corrected, and aligned to their respective $\mathrm{T} 1$ images. The first three volumes of EPI time series were removed because of T1-equilibrium effects, and the transformation matrix used to register the T1-weighted image to MNI space was applied to the functional data. Volume-to-volume displacement (SSD) was 
estimated from the six rigid body motion registration parameters. Any functional volume with SSD $>1 \mathrm{~mm}$ and its preceding volume were censored. Participants with $\geqslant 20 \%$ of volumes censored were excluded from analysis. Functional data were smoothed using a $6 \mathrm{~mm}$ Gaussian kernel. Final voxel size was $2 \times 2 \times 2 \mathrm{~mm}$.

\section{Statistical Analysis}

A first-level, within-subject model was constructed within AFNI 3dDeconvolve. The model included three blocks for each emotion condition, and the shape condition as fixedeffect regressors, modeled with a gamma hemodynamic response function of 1 -s duration. Six motion parameters and their derivatives were included as nuisance regressors, along with four polynomial drift terms. General linear tests were used to create emotion-shape contrasts for each emotion block that were then used in group analyses.

A second-level, between-subject model was constructed within AFNI 3dMVM (Chen et al, 2014). A $2 \times 2 \times 3$ mixed design included group (healthy, PTSD) as the betweensubject factor, and emotion (angry-shape, happy-shape) and block (1-3) as within-subject factors. Age was included as a covariate and interactions with the primary factors were examined, whereas sex was included as a nuisance covariate. Note that block was modeled to ascertain group differences in habituation/potentiation with repeated stimulus type presentation, as indicated in our prior study (Herringa et al, 2013b). Because we did not find any habituation/ potentiation effects by group, we averaged findings across block to facilitate presentation of findings. A priori search regions included bilateral $\mathrm{mPFC}$ and amygdala/hippocampus, using masks generated from AFNI standard template. The mPFC mask (Supplementary Figure S2) included the vmPFC (BA 10, 11, 25), dmPFC (BA 6, 8, 9), and ACC (BA 24, 32, 33). Multiple comparison correction was performed using Monte Carlo simulation in AFNI 3dClustSim (version April 2016). At a voxel-wise $p=0.01$, the corrected $\alpha=0.05$ cluster threshold was 141 voxels and 32 voxels for the mPFC and amygdala/hippocampus respectively. An additional Bonferroni correction for the use of two a priori search regions was applied (corrected $\alpha=0.025$ ). Additional results are reported outside of a priori search regions surviving whole-brain correction (cluster threshold 283 voxels).

Psychophysiological interaction (PPI) analysis was conducted within AFNI using seeds derived from our a priori search regions in the activation analysis. Voxel-wise interaction regressors were created using AFNI 3dSynthesize and 3 dTfitter, measuring the correlation between voxels in a given seed region with other voxels for each emotion condition versus shape. Interaction regressors and seed time series were entered as additional regressors in the first-level model from the activation analysis, for each seed. Group-level PPI analyses were conducted as described for the activation analysis. Given the exploratory nature of the PPI analyses, we did not apply additional Bonferroni correction for multiple seed regions to reduce the rate of false-negative results.

\section{Secondary Analyses}

Three multiple linear regression models were run on extracted cluster averages in SPSS v. 21 (IBM, Armonk, NY) to examine (1) potential confounds in group differences, (2) their relationships with PTSD, depression, and anxiety symptoms, and (3) their relationships with trauma exposure measures. Models (2) and (3) were performed within the PTSD group only. Group differences in task performance were also assessed within a separate linear model. All analyses were covaried for age and sex.

Given the high rates of comorbid affective and anxiety disorders in our sample, we used a transdiagnostic, dimensional symptom approach to examine the relationship between symptom measures and brain findings within the PTSD group as previously described (Patriat et al, 2016). This analysis is summarized here with additional details in Supplementary Material. Using a principal component analysis (PCA) of PTSD, depressive, and anxiety symptoms from the PTSD-RI, MFQ, and SCARED, we extracted five symptom dimension components explaining $74.8 \%$ of the total variance in symptom measures: social aversion, hopelessness, negative affect, hyperarousal, and re-experiencing.

\section{RESULTS}

\section{Participant Characteristics and Task Performance}

Participant characteristics are displayed in Table 1. The groups did not significantly differ in sex distribution, age, pubertal stage, IQ, or handedness. In addition, there were no group- or age-related differences on task performance. Please see Supplementary Material for further details.

\section{Regional Brain Activation}

Results in a priori search regions are summarized in Table 2. Additional results including whole brain findings are displayed in Supplementary Table 1. Note that trauma exposure measures (number of trauma types, time elapsed since index trauma, index trauma type) were not significant predictors of activation in the following results.

Within the amygdala and hippocampus, no PTSD main effects were present. However, a group by age interaction was observed in the right amygdala (extending into the anterior hippocampus; Figure 1a). Here, age was negatively correlated with activation in healthy youth, but positively correlated with activation in PTSD youth across emotion conditions $(r=-0.43$ and 0.55 , respectively). However, amygdala activation was not related to symptom severity in the PTSD group.

Within the $\mathrm{mPFC}$, a PTSD main effect in the right dmPFC (BA 9; Supplementary Figure S3) revealed greater activation, independent of emotion conditions, in PTSD compared with healthy youth. Furthermore, dmPFC activation was positively correlated with negative affect symptoms in the PTSD group (Table 2 and Supplementary Figure S3). In addition, a group by age interaction was present in the left rACC (BA 32; Supplementary Figure S3). Here, age was negatively correlated with activation in healthy youth, but positively correlated with activation in PTSD youth across emotion conditions ( $r=-0.65$ and 0.17 , respectively). Rostral ACC activation was also positively correlated with negative affect symptoms in the PTSD group (Table 2 and Supplementary Figure S3). Finally, a group by emotion interaction was observed in bilateral dACC (BA 24/32; Figure 1b). Surprisingly, PTSD youth showed hyperactivation to happy faces relative to 
Table I Participant Characteristics

\begin{tabular}{|c|c|c|}
\hline & Healthy & PTSD \\
\hline $\mathrm{N}$ & $28(15 \mathrm{~F})$ & $25(16 \mathrm{~F})$ \\
\hline Age (years) & $14.2( \pm 0.6)$ range: $8.13-18.46$ & | $4.3( \pm 0.6)$ range: $8.07-\mid 8.80$ \\
\hline Tanner stage & $3.3( \pm 0.2)$ range: $1-4.75$ & $3.3( \pm 0.3)$ range: $1-5$ \\
\hline IQ & $108.9( \pm 2.6)$ range: $81-133$ & $103.3( \pm 2.5)$ range: $77-125$ \\
\hline Left-handed $(n)$ & I & 3 \\
\hline Comorbid diagnoses (n) & - & $\begin{array}{l}\text { Major depressive disorder ( } 18) \text {, ADHD (5), social anxiety disorder (5), separation anxiety } \\
\text { disorder (3), generalized anxiety disorder (2), depressive disorder NOS (2) }\end{array}$ \\
\hline PTSD duration (months) & - & $42.0( \pm 7.7)$ \\
\hline PTSD Reaction Index & - & $42.8( \pm 2.5)$ \\
\hline CAPS-CA & - & $66.8( \pm 4.2)$ \\
\hline
\end{tabular}

Abbreviations: CAPS-CA, Clinician-Administered PTSD Scale for Children and Adolescents; MFQ, Mood and Feelings Questionnaire; SCARED, Screen for Child Anxiety-Related Emotional Disorders.

The healthy and PTSD groups did not significantly differ in sex distribution, age, Tanner stage, IQ, or handedness. The PTSD Reaction Index was determined by the greater of youth and caregiver scores for each item. CAPS-CA scores were not collected for the first four PTSD participants. The MFQ and SCARED represent the average of youth and caregiver reports. Numbers in parentheses with ' \pm ' represent SEM.

Table 2 Summary of Functional Activation Differences between PTSD and Healthy Youth in a Priori Search Regions of the mPFC and Amygdala/Hippocampus (Corrected $\alpha=0.025$ )

\begin{tabular}{|c|c|c|c|c|c|c|c|c|c|c|c|c|}
\hline Effect & Direction & Region & BA & $k$ & Volume $(\mu \mathrm{l})$ & Peak $F$ & $\mathbf{x}$ & $\mathbf{Y}$ & $\mathbf{Z}$ & $\begin{array}{l}\text { Post hoc regression } \\
\text { (PTSD only) }\end{array}$ & Std. $\beta$ & $P$ \\
\hline Group & PTSD > healthy & $\mathrm{R}$ dmPFC & 9 & 258 & 2064 & 13.32 & -6 & -40 & 24 & Negative affect & 2.761 & 0.018 \\
\hline Group $\times$ age & PTSD > healthy & $\mathrm{R}$ amygdala & - & 52 & 416 & 12.53 & -24 & 6 & -20 & - & - & - \\
\hline
\end{tabular}

Abbreviations: dACC, dorsal anterior cingulate cortex; dmPFC, dorsomedial prefrontal cortex; rACC, rostral anterior cingulate cortex.

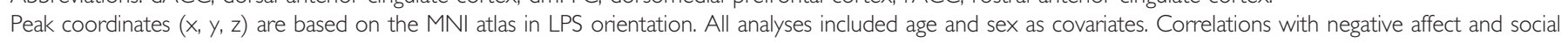
aversion symptoms were conducted in a multivariate regression including only youth with PTSD.

healthy youth, with no differences to angry faces. Dorsal ACC activation to happy faces was positively correlated with social aversion symptoms in the PTSD group, with no correlation for angry faces (Figure $1 \mathrm{~b}$ and Table 2).

\section{Functional Connectivity}

PPI results for the amygdala and AACC seeds are presented here given their central role in our hypotheses. PPI results for the rACC and dmPFC seeds are presented in Supplementary Material. All results from a priori search regions are summarized in Table 3. Additional results, including whole-brain findings, are displayed in Supplementary Table 2.

Right amygdala connectivity. Group by emotion interactions were present with bilateral dmPFC extending into the supplementary motor area (BA 9/8; Figure 2a), and left ventrolateral (vl) PFC/superior temporal gyrus (BA 47/38; Figure 2b). In each case, PTSD youth showed reduced connectivity to angry faces but increased connectivity to happy faces, the reverse pattern of healthy youth. AmygdaladmPFC and amygdala-vlPFC connectivity to happy faces were positively correlated with hyperarousal symptoms, with trending negative correlations to angry faces, within the PTSD group (Figures 2a and $\mathrm{b}$ ).

dACC connectivity. A group by emotion interaction was found with bilateral dmPFC (BA 9; Figure 2c). Here, PTSD youth again showed reduced connectivity to angry faces and increased connectivity to happy faces, the reverse pattern of healthy youth. Dorsal ACC-dmPFC connectivity was associated with trauma load: as the number of trauma types endorsed increased, dACC-dmPFC connectivity decreased 
a
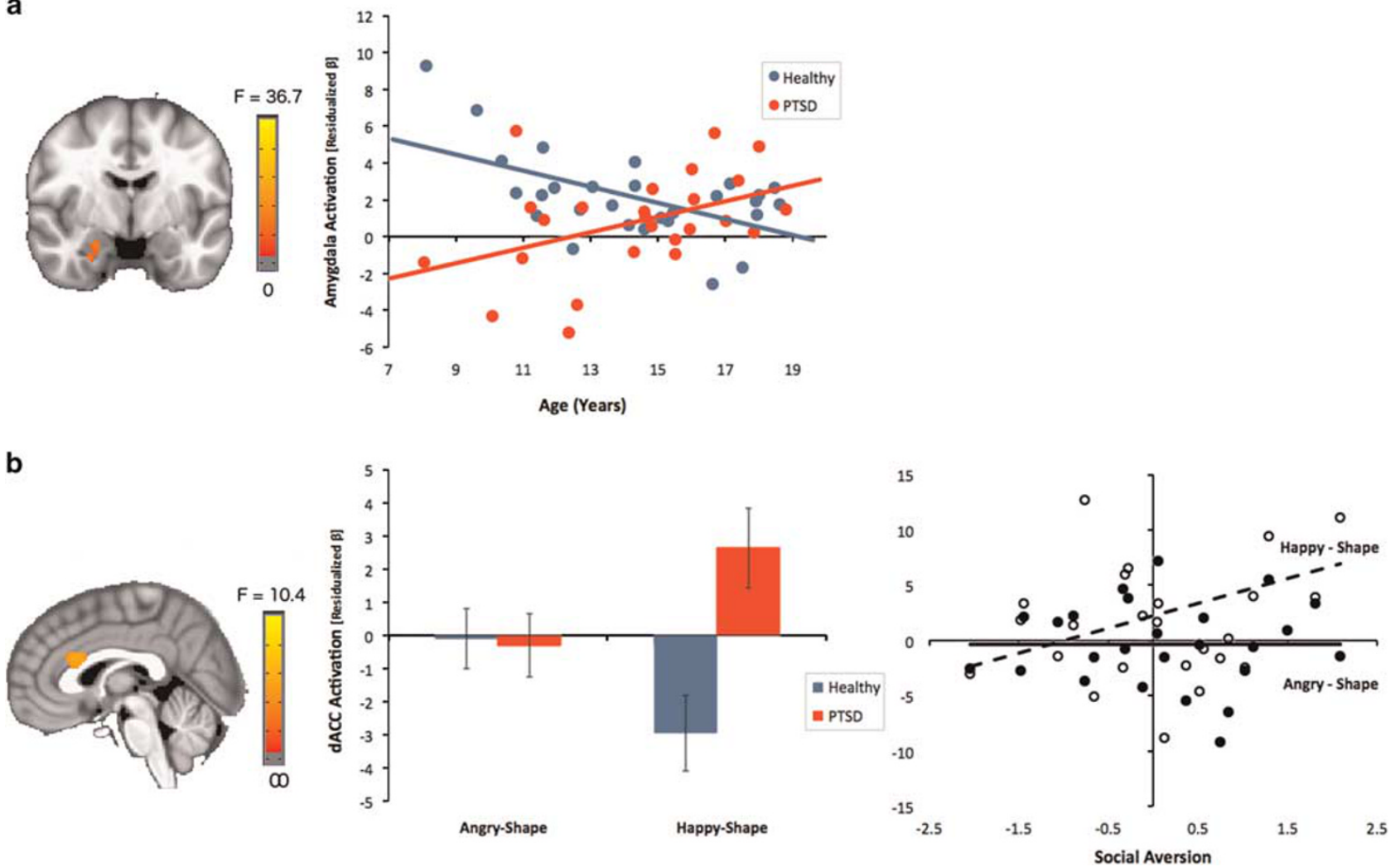

Figure I Regional brain activation abnormalities to dynamic emotional faces in pediatric PTSD. (a) A group by age interaction in right amygdala activation revealed decreases with age in healthy youth, but increased activation with age in PTSD youth across emotion conditions. Age-related findings remained when covaried for PTSD duration and time elapsed since index trauma. (b) Valence-abnormal brain activation to dynamic emotional faces in pediatric PTSD. A group by emotion interaction in the AACC (BA 24, 32) revealed hyperactivation in PTSD youth in the happy-shape contrast relative to healthy youth, with no group differences in the angry-shape contrast. Dorsal ACC activation in the happy-shape contrast was positively associated with social aversion symptoms within the PTSD cohort. All results were covaried for age and sex. Error bars indicate \pm I SEM. dACC $=$ dorsal anterior cingulate cortex.

in angry-shape and increased in happy-shape, reaching significance for $\geqslant 4$ trauma types endorsed (Figure $2 c$ ).

\section{Confound Analyses}

All results remained significant or trending $(p<0.1)$ when adjusted for potentially confounding variables. Please see Supplementary Material for further details.

\section{DISCUSSION}

To our knowledge, this is the first study to report age-related and valence-specific functional neural abnormalities during emotional face processing in pediatric PTSD. Consistent with our hypotheses, our study revealed increased amygdala activation with age in pediatric PTSD, suggesting a developmental sensitization of the amygdala to emotional stimuli. Unexpectedly, our study also revealed heightened recruitment of emotion appraisal and regulatory circuits to happy faces, and decreased recruitment to angry faces. These patterns are opposite to that of healthy youth and appear to differ from adult PTSD, where abnormal prefrontalamygdala function appears specific to negative emotional expressions. Importantly, valence-abnormal prefrontalamygdala recruitment was associated with social aversion and hyperarousal symptoms. Together, these findings point to abnormal salience and appraisal processing of emotional faces in pediatric PTSD that may directly contribute to dysregulated fear and anxiety in these youth.

Our findings indicate abnormal function and coupling in a network comprising the amygdala, dACC, and dmPFC. Dorso-rostral aspects of the $\mathrm{mPFC}$ have been heavily implicated in emotion appraisal and cognitive-emotional conflict regulation (Comte et al, 2016; Etkin et al, 2011; Kalisch et al, 2006; Kalisch and Gerlicher, 2014; Mechias et al, 2010). Notably, these regions contribute to high-level appraisal of emotional stimuli in a context-dependent manner (Kalisch and Gerlicher, 2014; Maren et al, 2013), including threat-safety discrimination (Lissek et al, 2014). Abnormal functioning of this network to threat stimuli has been implicated in pathological states of fear and anxiety (Etkin and Wager, 2007; Wolf and Herringa, 2016), putatively via enhanced amygdala responses (Mechias et al, 2010; Robinson et al, 2012).

Within this framework, we originally hypothesized that pediatric PTSD would be characterized by hyperactivation of emotion processing regions (amygdala, dACC) to threatrelated expressions, specifically angry faces. Adult PTSD studies (Dunkley et al, 2016; Felmingham et al, 2010; Fonzo et al, 2013) and studies of childhood trauma exposure (see, eg, Dannlowski et al, 2013; van Harmelen et al, 2013) 
suggest that this is indeed the case. However, our findings indicate that pediatric PTSD is characterized by relatively greater recruitment of emotion appraisal and conflict regulation circuits to happy faces, namely dACC hyperactivation, and increased amygdala-dmPFC and dACC-dmPFC connectivity. This suggests that youth with PTSD engage additional neural resources for successful task completion during happy faces, akin to processing of angry faces in healthy youth. Interestingly, amygdala activation itself did not show emotion-specific differential activation. Rather, valence-abnormal processing may begin with the dACC, the only node in the frontolimbic network to show emotionspecific activation differences.

The current findings extend those of our previous report in this sample examining prefrontal-amygdala function to threat images (Wolf and Herringa, 2016). In that study, we found dACC hyperactivation, accompanied by decreased amygdala-dmPFC connectivity, to threat images in PTSD youth. In contrast, the present study found dACC hyperactivation, accompanied by increased amygdala-dmPFC connectivity, to happy faces. One intriguing possibility is that youth with PTSD may engage similar implicit appraisal processes to threat images and happy faces. Here, dACC hyperactivation may reflect greater ambiguity of happy expressions with respect to threat. This notion is supported by the relationship between dACC activation and social aversion symptoms. At the same time, youth with PTSD may implicitly engage compensatory circuits, as suggested by increased amygdala-dmPFC coupling to happy faces, to allow successful task completion. On the other hand, the lack of dACC response to angry faces, combined with decreased amygdala-dmPFC coupling, may indicate insufficient recruitment of appraisal and regulatory resources to canonical threat expressions in pediatric PTSD. Together, these neural patterns suggest abnormal implicit threat-safety processing of emotional expressions, consistent with reports of abnormal facial emotion recognition in maltreated youth (Pollak et al, 2000; Pollak and Kistler, 2002; Pollak and Tolley-Schell, 2003).

Similarly, functional connectivity analyses revealed valence-abnormal connectivity between the dACC and dmPFC in pediatric PTSD. Here, youth with PTSD display increased dACC-dmPFC connectivity to happy faces, but decreased connectivity to angry faces. The dmPFC has been implicated in emotion appraisal, including the prediction of others' intentions based on facial information (Bzdok et al, 2013; Isoda and Noritake, 2013; Rushworth et al, 2013). Functional connectivity between the dACC and dmPFC has also been implicated in the evaluation of others' mental states (Li et al, 2014). In light of this, increased dACC-dmPFC connectivity to happy faces may reflect increased ambiguity in positive expressions, eliciting greater recruitment of regions involved in emotion appraisal.

Youth with PTSD also show increased amygdala-dmPFC and amygdala-vlPFC connectivity to happy faces, but decreased connectivity to angry faces. The amygdala, dmPFC, and vlPFC are key nodes in a network engaged during the evaluation of trustworthiness of other human faces (Bzdok et al, 2012), and their recruitment increases with cognitive-emotional conflict to emotional faces in healthy adults (Zaki et al, 2010). Furthermore, the dmPFC and vlPFC have been implicated in the regulation of 
a

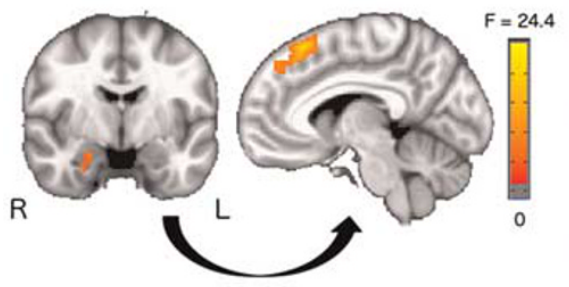

b

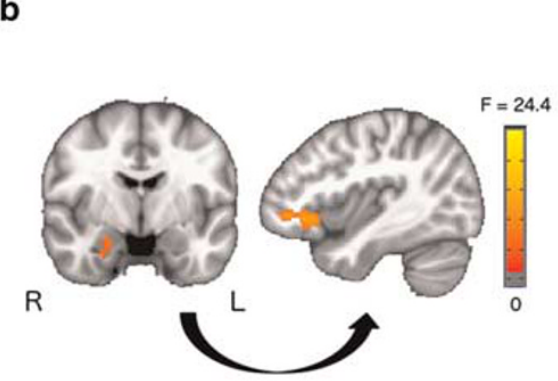

C

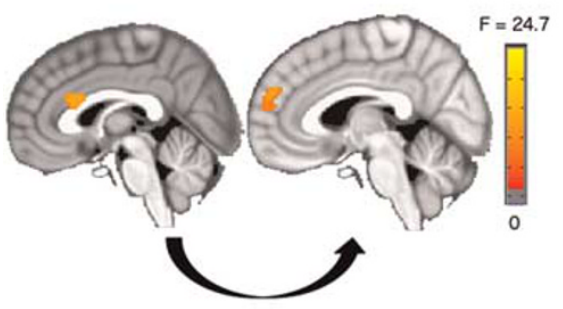

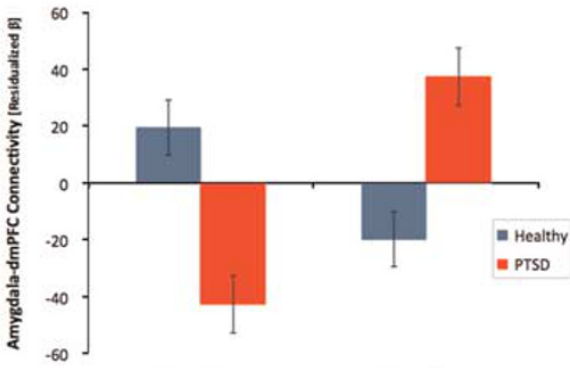

Angry-Shape

Happy-Shape

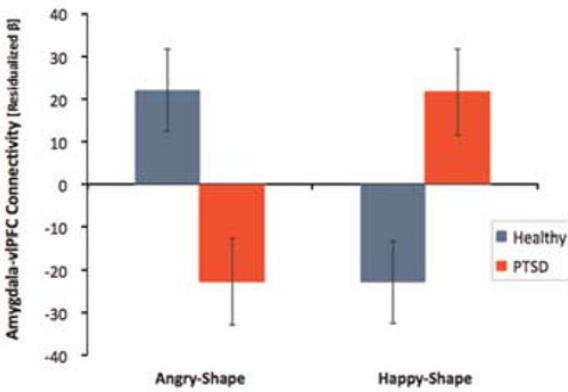

Angry-Shape

Happy-Shape

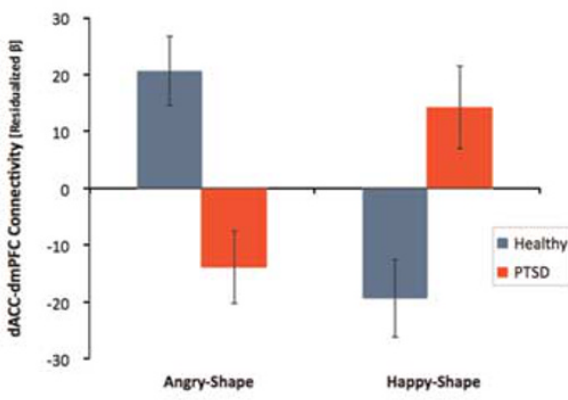

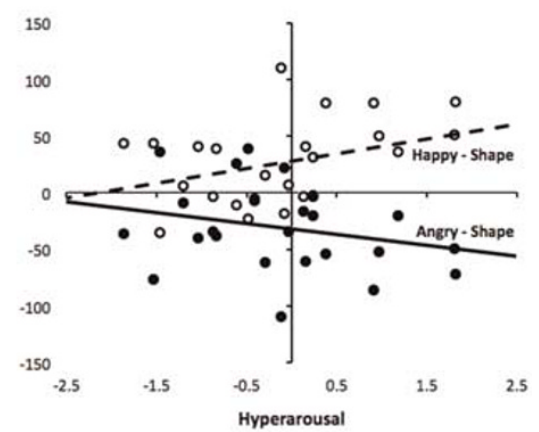

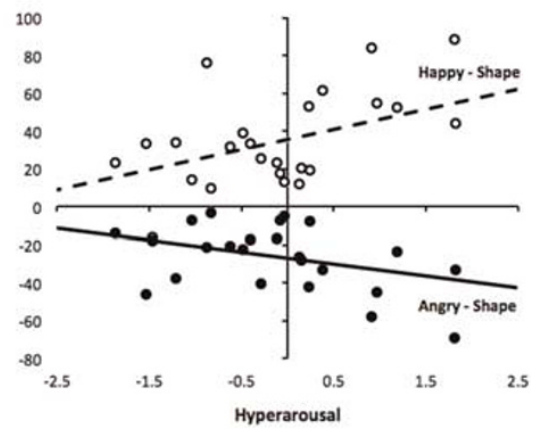

Hyperarousal

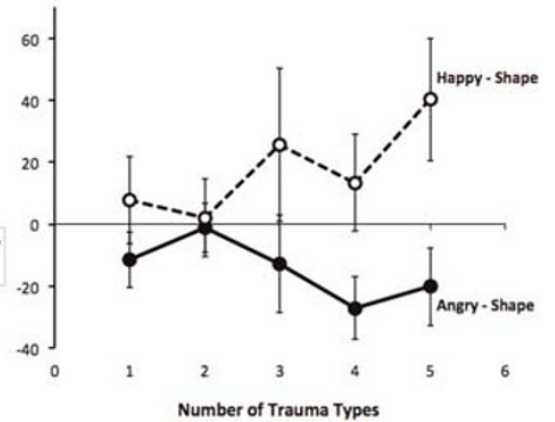

Figure 2 Valence-abnormal functional connectivity to dynamic emotional faces in pediatric PTSD. Seeds used in the connectivity analysis were derived from regional activation findings in the mPFC and amygdala/hippocampus. Group by emotion interactions were observed for functional connectivity in prefrontalamygdala pathways involved in emotion appraisal and regulation. In each case, PTSD youth showed decreased functional connectivity to angry faces, but increased connectivity to happy faces, the reverse pattern of healthy youth. (a) Right amygdala to bilateral dmPFC/SMA that was positively and negatively associated with hyperarousal symptoms in the happy-shape and angry-shape contrasts respectively; (b) right amygdala to left vIPFC/STG that was positively and negatively associated with hyperarousal symptoms in the happy-shape and angry-shape contrasts respectively; and (c) bilateral dACC to bilateral dmPFC that was positively and negatively associated with trauma type load in the happy-shape and angry-shape contrasts respectively. All results were covaried for age and sex. Error bars indicate \pm I SEM. dmPFC = dorsomedial prefrontal cortex; SMA = supplementary motor area; $\mathrm{dACC}=$ dorsal anterior cingulate cortex; $\mathrm{VIPFC}=$ ventromedial prefrontal cortex; STG = superior temporal gyrus.

emotional responses through their connections with the amygdala (Kim et al, 2013; Lee et al, 2012). Thus, it appears that pediatric PTSD is characterized by valence-inappropriate recruitment of emotion regulatory pathways while viewing happy and angry faces. Although this may allow for successful task completion, it may also reflect abnormal salience and appraisal processing of emotional faces, a notion supported by the association of these connectivity patterns with hyperarousal symptoms.

We find evidence of age-related abnormalities in amygdala function in pediatric PTSD that may indicate abnormal functional brain development. Consistent with prior studies in typically developing samples (Gee et al, 2013b; Vink et al, 2014), our healthy youth show decreased amygdala activation with age irrespective of face valence. In contrast, youth with PTSD show increased amygdala activation with age.
These age-related findings remained in the PTSD group when covarying for illness duration and time elapsed since index trauma, suggesting they are not secondary to these effects. Interestingly, amygdala activation was not related to symptom severity in the PTSD group, suggesting it may reflect a normative sensitization to face stimuli across development that could confer enhanced threat detection. Indeed, multiple studies have shown that childhood adversity and trauma are associated with amygdala hyperactivation by adulthood irrespective of symptoms (see, eg, Gee et al, 2013a; Herringa et al, 2016; Kim et al, 2013; McCrory et al, 2011; Swartz et al, 2015). Thus, although age-related sensitization of the amygdala may be present in youth who develop PTSD, the current study findings suggest that valence-abnormal processing in higher-level cortical regions may ultimately be responsible for the expression of PTSD. Future work, 
including treatment intervention designs, would be merited to explore these possibilities.

The results presented here show partial overlap with the few existing fMRI studies of pediatric PTSD/PTSS. Adolescents with PTSS show amygdala and mPFC hyperactivation to neutral and emotional faces (Garrett et al, 2012), and this is consistent with our findings of amygdala sensitization with age and dmPFC hyperactivation. In both studies, mPFC activation was positively related to symptom severity. On the other hand, Crozier et al (2014) reported sex differences in dmPFC activation, where maltreated males showed hyperactivation but maltreated females showed hypoactivation to fear faces. Surprisingly, other studies of pediatric PTSS/PTSD have not reported dACC hyperactivation (Crozier et al, 2014; Garrett et al, 2012; Yang et al, 2004) that we have observed in our sample across different emotion tasks (Wolf and Herringa, 2016). One possible reason for this is greater PTSD severity and comorbidity within the current sample, where other studies have recruited youth based on lower symptom thresholds. Finally, Cisler et al (2013) found a negative relationship between PTSD symptom severity and amygdala-dmPFC/pre-SMA connectivity to fearful faces in girls with a history of assault. This is perhaps one of the most consistent findings to date in pediatric PTSD/PTSS, with both the current study and our prior study (Wolf and Herringa, 2016) showing decreased amygdala-dmPFC connectivity to negative emotional stimuli, and further predicting PTSD severity.

The present study details novel findings regarding neural dysfunction in pediatric PTSD. It is not, however, without limitations. First, the lack of a healthy, trauma-exposed comparison group mitigates interpretation of results exclusively in light of PTSD and not trauma exposure per se. Yet, secondary analyses revealed differential relationships with trauma exposure and PTSD, with the benefit of examining these variables within subjects. Second, although our post hoc analyses suggest specificity of neural abnormalities to PTSD, anxiety, and depression symptom dimensions, we cannot determine diagnostic specificity given that PTSD status was the primary inclusion criterion in the clinical group. It is also important to note that PCA components were derived from a relatively small sample of youth and may not generalize to other study populations. Third, network abnormalities for PTSD main effects (with no valence effect) could reflect general face processing abnormalities. In addition, caution should be taken to avoid reverse inference errors with regard to fMRI. Although not confounded by task performance, we cannot definitively conclude whether neural abnormalities reflect compensation, state, or trait effects in our sample given the cross-sectional nature of the study. On the other hand, relating network abnormalities to a third variable, such as symptom severity, does allow for reasonable interpretation of functional abnormalities. Finally, it is possible that stimulus differences account for the apparent neural differences between adult and pediatric PTSD, given that many adult PTSD studies use fear and not angry faces.

In conclusion, the present study offers novel insights into the neural dysfunction associated with emotional face processing in pediatric PTSD. Our findings suggest that pediatric PTSD is characterized by paradoxical, valenceabnormal prefrontal-amygdala recruitment during emotional face processing. These neural patterns suggest that

youth with PTSD may require increased appraisal and regulatory resources for happy faces for successful task completion, akin to processing angry faces in healthy youth. These neural abnormalities suggest that positive emotional expressions may carry a greater degree of ambiguity and salience, whereas negative expressions have become reliably predictable for these afflicted youth. Although conceivably adaptive in abusive environments, abnormal face processing may come at the cost of poor or inefficient threat-safety discrimination, requiring heightened neural appraisal and regulatory resources in putatively safe contexts. Future studies would be merited to explore these findings longitudinally and assess whether successful treatment may restore normal functioning and development of this circuitry in pediatric PTSD.

\section{FUNDING AND DISCLOSURE}

The authors declare no conflict of interest.

\section{ACKNOWLEDGMENTS}

We thank Rachael Meline and Allison Blumenfeld who served as the study coordinators; Ariel Sherman, Erika Vassar-Olsen, Erin Long, Amanda Timek, and Shelby Weaver for assisting in study recruitment; Rick Wolf, Remi Patriat, and Dr Rasmus Birn for providing assistance with data analysis; and finally, Dr Richard Davidson for providing editorial comments on the manuscript. Funding for this study was provided by the National Institute of Mental Health Career Development Award (K08 MH100267, to $\mathrm{RJH})$, American Academy of Child and Adolescent Psychiatry Junior Investigator Award (to RJH), NARSAD Young Investigator Grant (to RJH), University of Wisconsin Institute for Clinical and Translational Research Translational Pilot Grant Award (NIH/NCATS UL1TR000427, to $\mathrm{RJH}$ ), and the University of Wisconsin School of Medicine and Public Health.

\section{REFERENCES}

Almeida JRC, de Kronhaus DM, Sibille EL, Langenecker SA, Versace A, Labarbara EJ et al (2011). Abnormal left-sided orbitomedial prefrontal cortical-amygdala connectivity during happy and fear face processing: a potential neural mechanism of female MDD. Front Psychiatry 2: 69.

Birmaher B, Khetarpal S, Brent D, Cully M, Balach L, Kaufman J et al (1997). The Screen for Child Anxiety Related Emotional Disorders (SCARED): scale construction and psychometric characteristics. J Am Acad Child Adolesc Psychiatry 36: 545-553.

Burghy CA, Stodola DE, Ruttle PL, Molloy EK, Armstrong JM, Oler JA et al (2012). Developmental pathways to amygdalaprefrontal function and internalizing symptoms in adolescence. Nat Neurosci 15: 1736-1741.

Bzdok D, Langner R, Hoffstaedter F, Turetsky BI, Zilles K, Eickhoff SB (2012). The modular neuroarchitecture of social judgments on faces. Cereb Cortex 22: 951-961.

Bzdok D, Langner R, Schilbach L, Engemann DA, Laird AR, Fox PT et al (2013). Segregation of the human medial prefrontal cortex in social cognition. Front Hum Neurosci 7: 232.

Chen G, Adleman NE, Saad ZS, Leibenluft E, Cox RW (2014). Applications of multivariate modeling to neuroimaging 
group analysis: a comprehensive alternative to univariate general linear model. Neuroimage 99: 571-588.

Cisler JM, Scott Steele J, Smitherman S, Lenow JK, Kilts CD (2013). Neural processing correlates of assaultive violence exposure and PTSD symptoms during implicit threat processing: a networklevel analysis among adolescent girls. Psychiatry Res 214: 238-246.

Cohen JA, Mannarino AP, Iyengar S (2011). Community treatment of posttraumatic stress disorder for children exposed to intimate partner violence: a randomized controlled trial. Arch Pediatr Adolesc Med 165: 16-21.

Cohen JA, Scheeringa MS (2009). Post-traumatic stress disorder diagnosis in children: challenges and promises. Dialogues Clin Neurosci 11: 91-99.

Comte M, Schön D, Coull JT, Reynaud E, Khalfa S, Belzeaux R et al (2016). Dissociating bottom-up and top-down mechanisms in the cortico-limbic system during emotion processing. Cereb Cortex 26: $144-155$

Costello EJ, Angold A (1988). Scales to assess child and adolescent depression: checklists, screens, and nets. J Am Acad Child Adolesc Psychiatry 27: 726-737.

Cox RW (1996). AFNI: software for analysis and visualization of functional magnetic resonance neuroimages. Comput Biomed Res 29: $162-173$.

Crozier JC, Wang L, Huettel SA, De Bellis MD (2014). Neural correlates of cognitive and affective processing in maltreated youth with posttraumatic stress symptoms: does gender matter? Dev Psychopathol 26: 491-513.

Dannlowski U, Kugel H, Huber F, Stuhrmann A, Redlich R, Grotegerd D et al (2013). Childhood maltreatment is associated with an automatic negative emotion processing bias in the amygdala. Hum Brain Mapp 34: 2899-2909.

Dunkley BT, Pang EW, Sedge PA, Jetly R, Doesburg SM, Taylor MJ (2016). Threatening faces induce fear circuitry hypersynchrony in soldiers with post-traumatic stress disorder. Heliyon 2: e00063.

Etkin A, Egner T, Kalisch R (2011). Emotional processing in anterior cingulate and medial prefrontal cortex. Trends Cogn Sci 15: 85-93.

Etkin A, Wager TD (2007). Functional neuroimaging of anxiety: a meta-analysis of emotional processing in PTSD, social anxiety disorder, and specific phobia. Am J Psychiatry 164: 1476-1488.

Felmingham K, Williams LM, Kemp AH, Liddell B, Falconer E, Peduto A et al (2010). Neural responses to masked fear faces: sex differences and trauma exposure in posttraumatic stress disorder. J Abnorm Psychol 119: 241-247.

Fonzo GA, Flagan TM, Sullivan S, Allard CB, Grimes EM, Simmons AN et al (2013). Neural functional and structural correlates of childhood maltreatment in women with intimatepartner violence-related posttraumatic stress disorder. Psychiatry Res 211: 93-103.

Fonzo GA, Simmons AN, Thorp SR, Norman SB, Paulus MP, Stein MB (2010). Exaggerated and disconnected insular-amygdalar blood oxygenation level-dependent response to threat-related emotional faces in women with intimate-partner violence posttraumatic stress disorder. Biol Psychiatry 68: 433-441.

Gabard-Durnam LJ, Flannery J, Goff B, Gee DG, Humphreys KL, Telzer E et al (2014). The development of human amygdala functional connectivity at rest from 4 to 23 years: a crosssectional study. Neuroimage 95: 193-207.

Garrett AS, Carrion V, Kletter H, Karchemskiy A, Weems CF, Reiss A (2012). Brain activation to facial expressions in youth with PTSD symptoms. Depress Anxiety 29: 449-459.

Gee DG, Gabard-Durnam LJ, Flannery J, Goff B, Humphreys KL, Telzer EH et al (2013a). Early developmental emergence of human amygdala-prefrontal connectivity after maternal deprivation. Proc Natl Acad Sci USA 110: 15638-15643.

Gee DG, Humphreys KL, Flannery J, Goff B, Telzer EH, Shapiro M et al (2013b). A developmental shift from positive to negative connectivity in human amygdala-prefrontal circuitry. J Neurosci 33: $4584-4593$.
Gilboa A, Shalev AY, Laor L, Lester H, Louzoun Y, Chisin R et al (2004). Functional connectivity of the prefrontal cortex and the amygdala in posttraumatic stress disorder. Biol Psychiatry 55: 263-272.

Hayes JP, Hayes SM, Mikedis AM (2012). Quantitative metaanalysis of neural activity in posttraumatic stress disorder. Biol Mood Anxiety Disord 2: 9.

Herringa RJ, Birn RM, Ruttle PL, Burghy CA, Stodola DE, Davidson RJ et al (2013a). Childhood maltreatment is associated with altered fear circuitry and increased internalizing symptoms by late adolescence. Proc Natl Acad Sci USA 110: 19119-19124.

Herringa RJ, Burghy CA, Stodola DE, Fox ME, Davidson RJ, Essex MJ (2016). Enhanced prefrontal-amygdala connectivity following childhood adversity as a protective mechanism against internalizing in adolescence. Biol Psychiatry Cog Neurosci Neuroimag 1: 326-334.

Herringa RJ, Phillips ML, Fournier JC, Kronhaus DM, Germain A (2013b). Childhood and adult trauma both correlate with dorsal anterior cingulate activation to threat in combat veterans. Psychol Med 43: 1533-1542.

Isoda M, Noritake A (2013). What makes the dorsomedial frontal cortex active during reading the mental states of others? Front Neurosci 7: 232.

Kalisch R, Gerlicher AMV (2014). Making a mountain out of a molehill: on the role of the rostral dorsal anterior cingulate and dorsomedial prefrontal cortex in conscious threat appraisal, catastrophizing, and worrying. Neurosci Biobehav Rev 42: 1-8.

Kalisch R, Wiech K, Critchley HD, Dolan RJ (2006). Levels of appraisal: a medial prefrontal role in high-level appraisal of emotional material. Neuroimage 30: 1458-1466.

Kaufman J, Birmaher B, Brent D, Rao U, Flynn C, Moreci P et al (1997). Schedule for Affective Disorders and Schizophrenia for School-Age Children-Present and Lifetime Version (K-SADS-PL): initial reliability and validity data. J Am Acad Child Adolesc Psychiatry 36: 980-988.

Keding TJ, Herringa RJ (2015). Abnormal structure of fear circuitry in pediatric post-traumatic stress disorder. Neuropsychopharmacology 40: 537-545.

Kim P, Evans GW, Angstadt M, Ho SS, Sripada CS, Swain JE et al (2013). Effects of childhood poverty and chronic stress on emotion regulatory brain function in adulthood. Proc Natl Acad Sci USA 110: 18442-18447.

Lee H, Heller AS, van Reekum CM, Nelson B, Davidson RJ (2012). Amygdala-prefrontal coupling underlies individual differences in emotion regulation. Neuroimage 62: 1575-1581.

Li W, Mai X, Liu C (2014). The default mode network and social understanding of others: what do brain connectivity studies tell us. Front Hum Neurosci 8: 74.

Lissek S, Bradford DE, Alvarez RP, Burton P, Espensen-Sturges T, Reynolds RC et al (2014). Neural substrates of classically conditioned fear-generalization in humans: a parametric fMRI study. Soc Cogn Affect Neurosci 9: 1134-1142.

Maren S, Phan KL, Liberzon I (2013). The contextual brain: implications for fear conditioning, extinction and psychopathology. Nat Rev Neurosci 14: 417-428.

McCrory EJ, De Brito SA, Sebastian CL, Mechelli A, Bird G, Kelly PA et al (2011). Heightened neural reactivity to threat in child victims of family violence. Curr Biol 21: R947-R948.

McLaughlin KA, Koenen KC, Hill ED, Petukhova M, Sampson NA, Zaslavsky AM et al (2013). Trauma exposure and posttraumatic stress disorder in a national sample of adolescents. J Am Acad Child Adolesc Psychiatry 52: 815-830 e14.

Mechias M-L, Etkin A, Kalisch R (2010). A meta-analysis of instructed fear studies: implications for conscious appraisal of threat. Neuroimage 49: 1760-1768.

Milad MR, Pitman RK, Ellis CB, Gold AL, Shin LM, Lasko NB et al (2009). Neurobiological basis of failure to recall extinction memory in posttraumatic stress disorder. Biol Psychiatry 66: 1075-1082. 
Patel R, Spreng RN, Shin LM, Girard TA (2012). Neurocircuitry models of posttraumatic stress disorder and beyond: a metaanalysis of functional neuroimaging studies. Neurosci Biobehav Rev 36: 2130-2142.

Patriat R, Birn RM, Keding TJ, Herringa RJ (2016). Default-mode network abnormalities in pediatric posttraumatic stress disorder. J Am Acad Child Adolesc Psychiatry 55: 319-327.

Pitman RK, Rasmusson AM, Koenen KC, Shin LM, Orr SP, Gilbertson MW et al (2012). Biological studies of post-traumatic stress disorder. Nat Rev Neurosci 13: 769-787.

Pollak SD, Cicchetti D, Hornung K, Reed A (2000). Recognizing emotion in faces: developmental effects of child abuse and neglect. Dev Psychol 36: 679-688.

Pollak SD, Kistler DJ (2002). Early experience is associated with the development of categorical representations for facial expressions of emotion. Proc Natl Acad Sci USA 99: 9072-9076.

Pollak SD, Tolley-Schell SA (2003). Selective attention to facial emotion in physically abused children. J Abnorm Psychol 112: 323-338.

Robinson OJ, Charney DR, Overstreet C, Vytal K, Grillon C (2012). The adaptive threat bias in anxiety: amygdala-dorsomedial prefrontal cortex coupling and aversive amplification. Neuroimage 60: 523-529.

Rushworth MFS, Mars RB, Sallet J (2013). Are there specialized circuits for social cognition and are they unique to humans? Curr Opin Neurobiol 23: 436-442.

St Jacques PL, Botzung A, Miles A, Rubin DC (2011). Functional neuroimaging of emotionally intense autobiographical memories in post-traumatic stress disorder. J Psychiatr Res 45: 630-637.

Steinberg AM, Brymer MJ, Decker KB, Pynoos RS (2004). The University of California at Los Angeles Post-traumatic Stress Disorder Reaction Index. Curr Psychiatry Rep 6: 96-100.
Stevens JS, Jovanovic T, Fani N, Ely TD, Glover EM, Bradley B et al (2013). Disrupted amygdala-prefrontal functional connectivity in civilian women with posttraumatic stress disorder. J Psychiatr Res 47: 1469-1478.

Swartz JR, Williamson DE, Hariri AR (2015). Developmental change in amygdala reactivity during adolescence: effects of family history of depression and stressful life events. Am J Psychiatry 172: 276-283.

van Harmelen $A L$, van Tol MJ, Demenescu LR, van der Wee NJ, Veltman DJ, Aleman A et al (2013). Enhanced amygdala reactivity to emotional faces in adults reporting childhood emotional maltreatment. Soc Cogn Affect Neurosci 8: 362-369.

Vink M, Derks JM, Hoogendam JM, Hillegers M, Kahn RS (2014). Functional differences in emotion processing during adolescence and early adulthood. Neuroimage 91: 70-76.

Weathers FW, Keane TM, Davidson JR (2001). Clinicianadministered PTSD scale: a review of the first ten years of research. Depress Anxiety 13: 132-156.

Wolf RC, Herringa RJ (2016). Prefrontal-amygdala dysregulation to threat in pediatric posttraumatic stress disorder. Neuropsychopharmacology 41: 822-831.

Woolrich MW, Jbabdi S, Patenaude B, Chappell M, Makni S, Behrens T et al (2009). Bayesian analysis of neuroimaging data in FSL. Neuroimage 45: S173-S186.

Yang P, Wu M-T, Hsu C-C, Ker J-H (2004). Evidence of early neurobiological alternations in adolescents with posttraumatic stress disorder: a functional MRI study. Neurosci Lett 370: 13-18.

Zaki J, Hennigan K, Weber J, Ochsner KN (2010). Social cognitive conflict resolution: contributions of domain general and domain specific neural systems. J Neurosci 30: 8481-8488.

Supplementary Information accompanies the paper on the Neuropsychopharmacology website (http://www.nature.com/npp) 